\title{
Nonequilibrium many-body quantum engine driven by time-translation symmetry breaking
}

\author{
Federico Carollo, ${ }^{1}$ Kay Brandner, ${ }^{2}$ and Igor Lesanovsky ${ }^{1,2}$ \\ ${ }^{1}$ Institut für Theoretische Physik, Universität Tübingen, \\ Auf der Morgenstelle 14, 72076 Tübingen, Germany \\ ${ }^{2}$ School of Physics and Astronomy and \\ Centre for the Mathematics and Theoretical Physics of Quantum Non-Equilibrium Systems, \\ University of Nottingham, Nottingham, NG7 2RD, UK
}

(Dated: October 15, 2020)

\begin{abstract}
Quantum many-body systems out of equilibrium can host intriguing phenomena such as transitions to exotic dynamical states. Although this emergent behaviour can be observed in experiments, its potential for technological applications is largely unexplored. Here, we investigate the impact of collective effects on quantum engines that extract mechanical work from a many-body system. Using an opto-mechanical cavity setup with an interacting atomic gas as a working fluid, we demonstrate theoretically that such engines produce work under periodic driving. The stationary cycle of the working fluid features nonequilibrium phase transitions, resulting in abrupt changes of the work output. Remarkably, we find that our many-body quantum engine operates even without periodic driving. This phenomenon occurs when its working fluid enters a phase that breaks continuous time-translation symmetry: the emergent time-crystalline phase can sustain the motion of a load generating mechanical work. Our findings pave the way for designing novel nonequilibrium quantum machines.
\end{abstract}

Future-generation nanomachines will require powerful small-scale engines whose energy output can be channeled into mechanical work storages. Proof-of-principle experiments have shown how such microscopic flywheels can be realized for working systems with few internal degrees of freedom like a single atom [1 15. Yet it is less clear how the output of a quantum engine can be converted into motive power if its working fluid consists of a many-body system.

During the last years, much progress has been made in the design of quantum engines that operate far from equilibrium and use non-thermal sources of energy [612. The natural next step is to explore how mechanical work can be generated in such non-equilibrium settings, how collective effects, like phase transitions, affect the work output and whether they could enable novel modes of operation.

In this article, we propose a new type of many-body quantum engine, that is driven by time-translation symmetry breaking [13-16] and does not require a periodic protocol. Our engine autonomously delivers mechanical work to an external load as a result of its working fluid hosting a phase with broken (continuous) timetranslation symmetry. Such a time-crystal phase features a time-dependent asymptotic state even in the absence of external modulation of the dynamical parameters [1316. We show that such an exotic device can be implemented with a general cavity-atom setting, which can, in principle, be realized in experiments with cold atoms 17 19, see the sketch in Fig 1. In this setup, one mirror of the cavity is fixed, while the other one is attached to a micro-spring and can move around its equilibrium position 2023 . By driving the atoms inside the cavity with a periodically modulated laser, the free mirror,

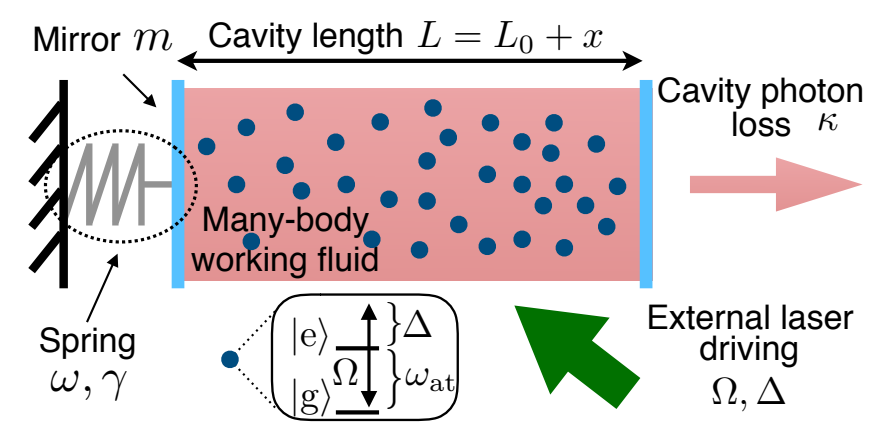

FIG. 1. Cavity-atom quantum engine. The atomic working fluid is held in an optical cavity with one movable mirror (mass $m$ ) attached to a spring (characteristic frequency $\omega$ ). The cavity length $L=L_{0}+x$ decomposes into an equilibrium length $L_{0}$ and a small deviation $x$. The motion of the mirror is damped by mechanical friction (proportional to the coefficient $\gamma$ ) and driven by thermal fluctuations and the radiation pressure inside the cavity. Each atom is described as a two-level system with ground state $|g\rangle$, excited state $|e\rangle$ and energy splitting $\omega_{\text {at }}$. Excitations are generated and destroyed through interactions with a light mode in the cavity and with the driving laser (Rabi frequency $\Omega$ and detuning $\Delta$ ). The cavity loss rate is $\kappa$.

which plays the role of a classical load, can be forced into sustained oscillations from which we determine the work delivered by the engine [24 30].

Our numerical analysis reveals two quite remarkable effects. First, the system features a series of nonequilibrium phase transitions leading to sudden changes of the asymptotic cycle. Second, even in the absence of an explicit periodic driving, where one would a priori expect the system to approach to a stationary state, the oscillatory motion of the mirror can be sustained as the atomic 
working fluid forms a time-crystalline phase [13 16] for properly chosen parameters.

Beyond illuminating these intriguing many-body effects, our approach has the key advantage that it admits a clear thermodynamic interpretation. Since the mirror is effectively classical, its position can be monitored without disturbing the operation cycle of the engine, thus avoiding the subtleties of measuring a quantum working fluid. Such quasi-classical work measurements make it possible to unambiguously determine the effective output of a quantum engine. At the same time, they open new ways to probe collective phenomena in nonequilibrium quantum many-body systems.

Cavity-atom setup. - We consider the setup of Fig.1. An ensemble of $N$ atoms is loaded into an optical cavity with one movable mirror. Each atom is described as a two-level system with ground state $|\mathrm{g}\rangle$, excited state $|\mathrm{e}\rangle$, and level splitting $\omega_{\text {at }}$. A single light mode is resonant with the cavity at frequency $\omega_{\text {cav }}$. The exchange of photons between atoms and light field is described by the coupling Hamiltonian

$$
H_{\mathrm{int}}=\hbar \frac{g}{\sqrt{N}}\left(a S_{+}+a^{\dagger} S_{-}\right) \quad \text { with } \quad S_{ \pm}=\sum_{k=1}^{N} \sigma_{ \pm}^{(k)} .
$$

Here, $a$ and $a^{\dagger}$ are the photon creation and annihilation operators and $\sigma_{-}=|\mathrm{g}\rangle\langle\mathrm{e}|$ and $\sigma_{+}=\sigma_{-}^{\dagger}$ are the atomic transition operators. The interaction strength is rescaled by the factor $1 / \sqrt{N}$ as is common for light-matter interactions of this type [31 33]. The atoms are further driven by an external laser, whose frequency is shifted from $\omega_{\text {at }}$ by the detuning $\Delta$. In the rotating frame of the laser, the atomic Hamiltonian is given by [19, 34 36 .

$$
H_{\mathrm{L}}=\hbar\left[\Omega\left(S_{+}+S_{-}\right)-\frac{\Delta}{2} S_{z}\right] \quad \text { with } \quad S_{z}=\sum_{k=1}^{N} \sigma_{z}^{(k)}
$$

where $\sigma_{z}=|\mathrm{e}\rangle\langle\mathrm{e}|-| \mathrm{g}\rangle\langle\mathrm{g}|$. The Rabi frequency $\Omega$ is determined by the strength of the coherent driving. In the same rotating frame, the free Hamiltonian of the light field reads $H_{\mathrm{ph}}=-\hbar \delta a^{\dagger} a$, where $\delta=\omega_{\text {at }}+\Delta-\omega_{\text {cav }}$ is the effective detuning of the cavity mode. The loss of photons from the cavity, at rate $\kappa$, is described by the dissipation super operator $37-40$.

$$
\mathcal{D}_{\mathrm{ph}}[\rho]=\hbar \kappa\left(a \rho a^{\dagger}-\frac{1}{2}\left\{\rho, a^{\dagger} a\right\}\right) .
$$

In the Schrödinger picture, the bare photon Hamiltonian is given by $H_{\mathrm{ph}}^{\mathrm{S}}=\hbar \omega_{\text {cav }} a^{\dagger} a$. The frequency of the photons is connected to the length $L$ of the cavity through the resonance condition $\omega_{\text {cav }}=n c /(2 L)$ with $n$ being an integer and $c$ the speed of light. We decompose the length of the cavity, $L=L_{0}+x$, into an equilibrium contribution $L_{0}$ and a deviation $x$, which accounts for small oscillations of the first mirror, see Fig. 1. Expanding the Hamiltonian $H_{\mathrm{ph}}^{\mathrm{S}}$ to first order in $x / L_{0}$ yields [20 22]

$$
H_{\mathrm{ph}}^{\mathrm{S}} \approx \hbar \omega_{\mathrm{cav}}^{0}\left(1-\frac{x}{L_{0}}\right) a^{\dagger} a \quad \text { with } \quad \omega_{\mathrm{cav}}^{0}=\frac{n c}{2 L_{0}} .
$$

This result shows that the position of the mirror and the number of photons are coupled [20 22, 29]. In fact, the Hamiltonian in Eq. (3) describes a mechanical force on the mirror, which emerges from the radiation pressure inside the cavity.

In addition, the light field in the cavity mediates an effective excitation-exchange coupling between the atoms, which arises from the interaction Hamiltonian (1) when the electromagnetic field is traced out [41, 42]. In the weak-coupling regime $\kappa \gg g / \sqrt{N}$, the state of the atoms $\rho_{t}$ follows an effective Lindblad equation [18, 37, 40, 43.

$$
\dot{\rho}_{t}=-\frac{i}{\hbar}\left[\tilde{H}, \rho_{t}\right]+\frac{1}{\hbar} \tilde{\mathcal{D}}\left[\rho_{t}\right] .
$$

Upon neglecting second-order contributions in the relative displacement $x / L_{0}$, the corresponding effective Hamiltonian and dissipation super-operator become

$$
\tilde{H}=H_{\mathrm{L}}+\frac{\hbar g}{N}\left(C_{0}-\frac{C_{1}}{L_{0}} x\right) S_{+} S_{-},
$$

and

$$
\tilde{\mathcal{D}}[\rho]=\frac{\hbar g}{N}\left(\Gamma_{0}-\frac{\Gamma_{1}}{L_{0}} x\right)\left(S_{-} \rho S_{+}-\frac{1}{2}\left\{\rho, S_{+} S_{-}\right\}\right),
$$

with the dimensionless constants [43]

$$
\begin{array}{rr}
C_{0}=\frac{4 \delta_{0} g}{\kappa^{2}+4 \delta_{0}^{2}}, & \Gamma_{0}=\frac{4 \kappa g}{\kappa^{2}+4 \delta_{0}^{2}} \\
C_{1}=\frac{\omega_{\mathrm{cav}}^{0}}{\delta_{0}} C_{0} \frac{4 \delta_{0}^{2}-\kappa^{2}}{4 \delta_{0}^{2}+\kappa^{2}}, & \Gamma_{1}=\omega_{\mathrm{cav}}^{0} \Gamma_{0} \frac{8 \delta_{0}}{\kappa^{2}+4 \delta_{0}^{2}}
\end{array}
$$

and the detuning parameter $\delta_{0}=\omega_{\mathrm{at}}+\Delta-\omega_{\mathrm{cav}}^{0}$.

Dynamics of the mirror.- The mirror is a massive object, whose ground state energy is small compared to the typical energy of thermal excitations. That is, we have $\hbar \omega \ll k_{\mathrm{B}} T$, where $\omega$ is the characteristic frequency of the spring attached to the mirror, see Fig. 1 and $T$ is the base temperature of the setup. The position of the mirror can thus be treated as a classical degree of freedom, whose dynamics is governed by the Langevin equation [27, 44]

$$
m \ddot{x}+\gamma \dot{x}+m \omega^{2} x=f_{t}+\xi_{t} ;
$$

here, $\gamma$ is the damping coefficient, $m$ is the mass of the mirror and the stochastic force $\xi_{t}$, which describes 


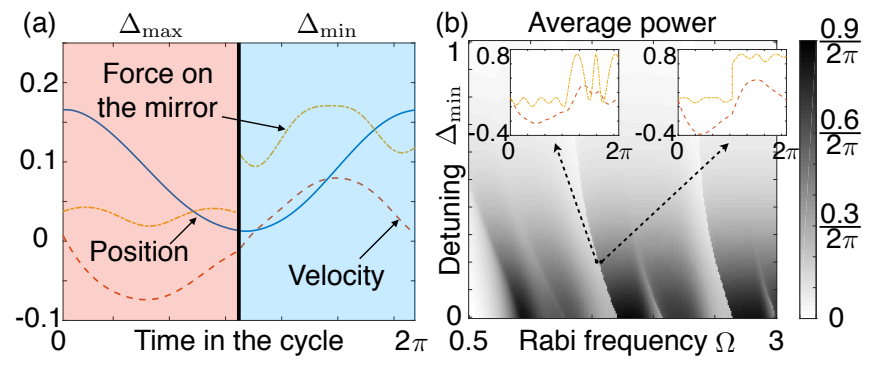

FIG. 2. Periodic driving. (a) Periodic motion of the mirror driven by the many-body engine. The average velocity of the mirror, $\bar{v}_{t}$, is given in units of $\mathrm{v}_{0}=\frac{\omega_{\mathrm{cav}}^{0}}{\omega} \frac{\hbar D_{0}}{m}$, the average position, $\bar{x}_{t}$, in units of $\mathrm{v}_{0} / \omega$ and the force in units of $\hbar \omega_{\text {cav }}^{0} D_{0}$. Time is given in units of $\omega^{-1}$. This representative cycle is obtained for $\omega_{\text {at }}-\omega_{\text {cav }}^{0}=0.1 \omega, \Delta_{\max }=2 \omega$ and $\Delta_{\min }=\kappa=g=\omega$. The velocity, averaged over noise realizations, $\bar{v}_{t}$ is not always zero during the cycle, proving that the engine constantly delivers energy to the mirror through the force $f_{t}$. (b) Power output, in units of $m \mathrm{v}_{0}^{2} \omega$, as a function of $\Omega / \omega$ and $\Delta_{\min } / \omega$ with fixed $\Delta_{\max }-\Delta_{\min }=\omega$. In the insets, we show the mean velocity and the force for $\Delta_{\min }=0.2 \omega$ and two slightly different values of the Rabi frequency, $\Omega / \omega=1.55,1.56$, for which the power output differs substantially. The force profile changes abruptly from an oscillatory pattern to a two plateau-like shape indicating a non-equilibrium phase transition. Numerical results have been obtained by simulating the dynamics of the mirror for sufficiently long times, such that the system has converged to its asymptotic cycle.

thermal fluctuations, obeys $\mathbb{E}\left[\xi_{t}\right]=0$ and $\mathbb{E}\left[\xi_{t} \xi_{s}\right]=$ $2 \gamma k_{\mathrm{B}} T \delta(t-s)$. The symbol $\mathbb{E}$ indicates average over all realizations [45].

The deterministic force $f_{t}=-\left\langle\left[\frac{\partial}{\partial x} H_{\mathrm{ph}}^{\mathrm{S}}\right]\right\rangle_{t}$ is due to the light-mediated coupling between the mirror and the working fluid. In the effective picture of an interacting atomic gas, it is, up to second-order corrections in the displacement of the mirror $x / L_{0}$, given by [43,

$$
f_{t} \approx \hbar \frac{g}{N} \frac{\omega_{\mathrm{cav}}^{0}}{\kappa L_{0}}\left(\Gamma_{0}-\Gamma_{1} \frac{x}{L_{0}}\right)\left\langle S_{+} S_{-}\right\rangle_{t},
$$

where angular brackets denote the average with respect to the state of the atomic system $\rho_{t}$. Together with this relation, the effective master equation (4) and the Langevin equation (7) determine the joint dynamics of the mirror and the working fluid.

Finite-density limit. - We now consider the limit of large atom numbers, $N \gg 1$, focussing on the case where the linear density of atoms in the cavity, $D_{0}=N / L_{0}$, is fixed. This assumption, which is typically well justified in experiments, makes it possible to simplify our mathematical model. First, the constants $C_{1}$ and $\Gamma_{1}$, appearing in Eqs. (5)-(6), become irrelevant for the dynamics, as they are of order $N^{-2}$, and can thus be neglected. Second, the normalized correlation functions $\left\langle S_{+} S_{-}\right\rangle / N^{2}$ factorize, since emergent correlations between different atoms are wiped out in the large- $N$ limit [46, 47]. That is, we have $\left\langle S_{+} S_{+}\right\rangle / N^{2} \sim s_{+} s_{-}$with $s_{ \pm}=\lim _{N \rightarrow \infty}\left\langle S_{ \pm}\right\rangle / N$.

As a result, the collective atomic variables $s_{ \pm}$and $s_{z}=\lim _{N \rightarrow \infty}\left\langle S_{z}\right\rangle / N$ obey the mean-field type dynamical equations [43, 47.

$$
\begin{aligned}
\dot{s}_{+} & =-i \Omega s_{z}-i \Delta s_{+}-i g C_{0} s_{z} s_{+}+\frac{g_{0} \Gamma_{0}}{2} s_{z} s_{+}, \\
\dot{s}_{z} & =2 i \Omega\left(s_{-}-s_{+}\right)-2 g \Gamma_{0} s_{+} s_{-},
\end{aligned}
$$

and the Langevin equation (7) becomes

$$
m \ddot{x}+\gamma \dot{x}+m \omega^{2} x=\hbar \frac{g \omega_{\mathrm{cav}}^{0} \Gamma_{0} D_{0}}{\kappa} s_{+} s_{-}+\xi_{t} ;
$$

since the expression (8) for the deterministic force reduces to

$$
f_{t}=\hbar \frac{g \omega_{\mathrm{cav}}^{0} \Gamma_{0} D_{0}}{\kappa} s_{+} s_{-}
$$

in the thermodynamic limit. The Eqs. $\sqrt{9}-(10)$ provide a complete dynamical model of our engine in terms of the four variables $s_{ \pm}, s_{z}$ and $x$.

Work extraction through the mirror.- We first consider a conventional isothermal engine cycle, where the detuning $\Delta$ is periodically modulated to provide energy input. For simplicity, we focus on a quench protocol, where $\Delta=\Delta_{\max }$ during the first half of the period and $\Delta=\Delta_{\text {min }}$ during the second half, as shown in Fig. 2(a). The period $t_{\mathrm{c}}$ of the driving is resonant with the eigenfrequency of the mirror, $t_{\mathrm{c}}=2 \pi / \omega$. As a consequence of the driving, the state of the atoms $\rho_{t}$ and the average position of the mirror approach an asymptotic cycle with period $t_{\mathrm{c}}$. As initial conditions we consider all atoms in their ground state and the mirror in its equilibrium position $\left(x_{0}=\dot{x}_{0}=0\right)$. However, at least at a qualitative level, our results do not depend on the specific initial conditions.

Owing to energy conservation, the average amount of work that is transferred from the atomic system to the mirror plus the energy contribution of the thermal fluctuations must be equal to the average heat dissipation due to mechanical friction. Hence, the power delivered by the engine per cycle is given by

$$
P_{\mathrm{av}}=\frac{\gamma}{t_{\mathrm{c}}} \int_{0}^{t_{\mathrm{c}}} d t\left(\mathbb{E}\left[v_{t}^{2}\right]-\frac{k_{\mathrm{B}} T}{m}\right)=\frac{\gamma}{t_{\mathrm{c}}} \int_{0}^{t_{\mathrm{c}}} d t \bar{v}_{t}^{2},
$$

as shown in [43, together with a thermodynamic description of our engine, by using tools of stochastic thermodynamics. Here, $v_{t}=\dot{x}_{t}$ is the velocity of the mirror, $\gamma k_{\mathrm{B}} T / m$ represents the thermal energy dissipated by mechanical friction, and $\bar{v}_{t}$ is the velocity of the mirror averaged over noise realizations - which can be obtained from Eq. (10) with $\xi_{t}=0$.

In Fig. 2(b), the generated mean power $P_{\mathrm{av}}$ is plotted as a function of the Rabi frequency $\Omega$ and the lower level 
of the detuning $\Delta_{\min }$. We find that $P_{\mathrm{av}}$ is positive over a large range of parameters. This result proves that our engine is able to produce usable work by sustaining the periodic motion of the mirror against constant damping. Quite remarkably, the average power output features discontinuous jumps signalling nonequilibrium phase transitions in the asymptotic periodic state as illustrated by the insets of Fig. 2(b). This new type of phase transition generalizes steady-state nonequilibrium ones to periodically driven settings. The power output acts as an order parameter which can be used to unveil the occurence of sudden changes in the asymptotic periodic dynamics of the many-body working fluid.

Time-translation symmetry breaking.- Once the time-dependent modulation of the detuning is turned off, one would expect the mirror to come to rest as the working fluid settles to a steady state. However, our analysis shows that, for properly chosen parameters, the engine still drives sustained oscillations of the mirror, even if the detuning is fixed. This a priori surprising phenomenon arises as a consequence of the working fluid entering a dissipative time-crystal phase, which breaks the (continuous) temporal translation symmetry of the generator. This exotic phase emerges when the asymptotic atom state is time dependent, despite the dynamical generator being time independent [15, 16]. The engine thereby acquires a new operation mechanism, which does not require cyclic control protocols and instead makes it possible to generate periodic motion from steady-state driving, as illustrated in Fig. 3(a).

In the absence of a periodic protocol, there is no natural recurrence time for the long-time dynamics which, in general, may or may not approach an asymptotic cycle. To explore this regime quantitatively, we thus need to determine the average power of the engine by calculating the average heat loss generated by the mirror over a long time window. Namely, we compute the power as

$$
P_{\mathrm{av}}=\lim _{t_{\mathrm{obs}} \rightarrow \infty} \frac{\gamma}{t_{\mathrm{obs}}} \int_{0}^{t_{\mathrm{obs}}} d t \bar{v}_{t}^{2} .
$$

If the position of the mirror settles on an asymptotic cycle with a well-defined period, this definition coincides with the one given in Eq. 12 .

The results of our analysis are summarized in Fig. 3.(b). In the weak photon-loss regime, i.e. for $\kappa \ll \Omega$, the working fluid settles to a stationary state where no mechanical work is produced. Approximately at $\kappa \sim 1 / \Omega$, for the specific choice of parameters, a phase transition occurs and the average power abruptly increases as the mirror breaks into sustained oscillations. This effect is most pronounced at moderate photon-loss rates, where our timecrystal engine delivers the largest output.

The working mechanism of this new operation mode of the engine can be understood as follows. The effective
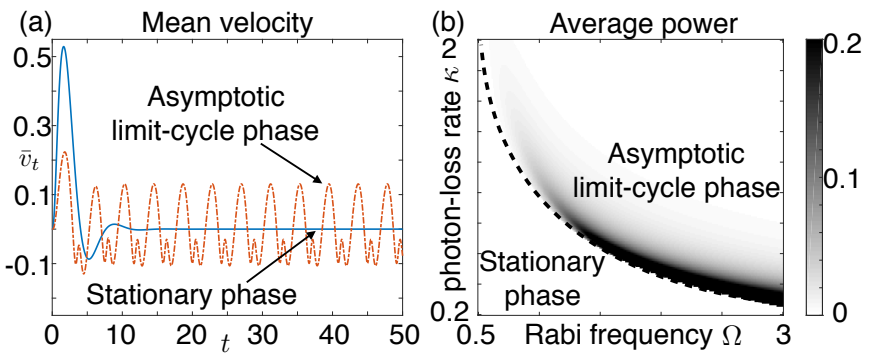

FIG. 3. Time-crystal quantum engine.

(a) Mean ve-

locity of the mirror in units of $\mathrm{v}_{0}=\frac{\omega_{\text {cav }}^{0}}{\omega} \frac{\hbar D_{0}}{m}$ as a function of time (in units of $\omega^{-1}$ ) for two different photon-loss rates. For $\kappa / \omega=0.5$, the mirror comes to rest while sustained oscillations emerge for $\kappa / \omega=1.5$. For this plot we have set $\Delta=0$, $\omega_{\text {at }}-\omega_{\text {cav }}^{0}=0.1 \omega$ and $\Omega=g=\omega$. (b) Average power output, in units of $m \mathrm{v}_{0}^{2} \omega$, as a function of the photon-loss rate $\kappa$ and the Rabi frequency $\Omega$, both in units of $\omega$. The remaining parameters are the same as before. Along the dashed line $\kappa \Omega \sim 1$, a phase transition occurs, where the average power jumps to a finite value as the working fluid spontaneously forms a time crystal. The scale has been truncated at 0.2 , but significantly larger values for the work output $\left(P_{\mathrm{av}}>2\right)$ are found. The maximum value, for the chosen parameters, is given by $P_{\mathrm{av}} \sim 2.8$.

dissipation constant $\Gamma_{0}$ decays with large photon-loss rates $\kappa$. In this regime, characterized by weak dissipation on the atoms $\left(\Gamma_{0} \ll 1\right)$, the dynamics of the working fluid is dominated by the Rabi driving at frequency $\Omega$. As a result, the steady-state manifold of the atomic system becomes degenerate and long-lived oscillations within this manifold emerge [15, 16]. The asymptotic state of the system is therefore time dependent and generates coherent oscillations leading to a varying force on the mirror. When atomic dissipation dominates, coherent oscillations are suppressed and the working fluid approaches a time-invariant steady-state. In this case, the radiation pressure on the mirror is constant and the mirror comes to rest.

Discussion.- We have developed a general framework for the dynamical description of many-body quantum engines, which includes the external load as a semi-classical degree of freedom. This approach makes it possible to determine the performance of the engine directly by monitoring the coupled dynamics of both the working fluid and of the load.

This perspective allowed us to obtain two key results. First, in the periodic mode of operation, where the engine is driven by modulations of an external control parameter, a new type of nonequilibrium phase transitions emerges. The power output of the engine thereby plays the role of an order parameter.

Second, we have demonstrated that, even when the engine is not driven through a periodic protocol, and is thus described by a time-independent generator, it can 
still deliver mechanical work. The emergence of this new regime is due to a nonequilibrium phase transition in the atomic working fluid towards an exotic state that features sustained coherent oscillations. This dynamical time-crystalline phase 13 16 can drive the motion of the load without relying on a time-dependent control protocol. Our approach paves the way to explore new mechanisms of power generation enabled by collective manybody effects and, at the same time, provides a natural description of many-body quantum engines.

Our predictions on the power output of our engine can be tested with current technology in cavity-atom experiments [17, 18, 48, 52, and our general numerical analysis covers a wide range of different setups. As we show in Ref. [43], the broken time-translation symmetry regime is robust to the presence of additional atomic interactions of density-density type and of excitation-exchange type. However, in the presence of completely generic perturbations we expect the time-translation symmetry to be restored at long times, and a survival of the time-crystal quantum engine only as a metastable regime. Still power generation for the periodic driving case is stable against any type of perturbations.

Finally, we note that, for the finite-density case that we have considered, the efficiency of our engine is effectively zero in the thermodynamic limit 43 . For finite systems -or even for different thermodynamic limits keeping $L_{0}$ finite - the efficiency can be larger than zero. However, even in the regime considered here the engine delivers finite power.

FC acknowledges support through a Teach@Tübingen Fellowship. KB received support from the University of Nottingham through a Nottingham Research Fellowship and from UK Research and Innovation through a Future Leaders Fellowship (Grant Reference: MR/S034714/1). IL acknowledges support from the DFG through SPP 1929 (GiRyd) under Project No. 428276754, and under Project No. 390727645, as well as from the "Wissenschaftler-Rückkehrprogramm GSO/CZS" of the Carl-Zeiss-Stiftung and the German Scholars Organization e.V.

[1] Holger Thierschmann, Rafael Sánchez, Björn Sothmann, Fabian Arnold, Christian Heyn, Wolfgang Hansen, Hartmut Buhmann, and Laurens W. Molenkamp, "Threeterminal energy harvester with coupled quantum dots," Nature Nanotechnology 10, 854-858 (2015).

[2] Johannes Roßnagel, Samuel T. Dawkins, Karl N. Tolazzi, Obinna Abah, Eric Lutz, Ferdinand Schmidt-Kaler, and Kilian Singer, "A single-atom heat engine," Science 352, 325 (2016)

[3] Martin Josefsson, Artis Svilans, Adam M. Burke, Eric A. Hoffmann, Sofia Fahlvik, Claes Thelander, Martin Leijnse, and Heiner Linke, "A quantum-dot heat engine operating close to the thermodynamic efficiency limits,"
Nature Nanotechnology 13, 920-924 (2018).

[4] D. von Lindenfels, O. Gräb, C. T. Schmiegelow, V. Kaushal, J. Schulz, Mark T. Mitchison, John Goold, F. Schmidt-Kaler, and U. G. Poschinger, "Spin heat engine coupled to a harmonic-oscillator flywheel," Phys. Rev. Lett. 123, 080602 (2019).

[5] John P. S. Peterson, Tiago B. Batalhão, Marcela Herrera, Alexandre M. Souza, Roberto S. Sarthour, Ivan S. Oliveira, and Roberto M. Serra, "Experimental characterization of a spin quantum heat engine," Phys. Rev. Lett. 123, 240601 (2019)

[6] Cyril Elouard, David Herrera-Martí, Benjamin Huard, and Alexia Auffèves, "Extracting work from quantum measurement in Maxwell's Demon engines," Phys. Rev. Lett. 118, 260603 (2017)

[7] Cyril Elouard and Andrew N. Jordan, "Efficient quantum measurement engines," Phys. Rev. Lett. 120, 260601 (2018)

[8] Wolfgang Niedenzu and Gershon Kurizki, "Cooperative many-body enhancement of quantum thermal machine power," New Journal of Physics 20, 113038 (2018).

[9] Marco Pezzutto, Mauro Paternostro, and Yasser Omar, "An out-of-equilibrium non-Markovian quantum heat engine," Quantum Science and Technology 4, 025002 (2019)

[10] Nicole Yunger Halpern, Christopher David White, Sarang Gopalakrishnan, and Gil Refael, "Quantum engine based on many-body localization," Phys. Rev. B 99, 024203 (2019)

[11] Najmeh Etehadi Abari, Giulia Vittoria De Angelis, Stefano Zippilli, and David Vitali, "An optomechanical heat engine with feedback-controlled in-loop light," New Journal of Physics 21, 093051 (2019)

[12] Federico Carollo, Filippo M. Gambetta, Kay Brandner, Juan P. Garrahan, and Igor Lesanovsky, "Nonequilibrium quantum many-body Rydberg atom engine," Phys. Rev. Lett. 124, 170602 (2020).

[13] Frank Wilczek, "Quantum time crystals," Phys. Rev. Lett. 109, 160401 (2012)

[14] Alfred Shapere and Frank Wilczek, "Classical time crystals," Phys. Rev. Lett. 109, 160402 (2012).

[15] F. Iemini, A. Russomanno, J. Keeling, M. Schirò, M. Dalmonte, and R. Fazio, "Boundary time crystals," Phys. Rev. Lett. 121, 035301 (2018)

[16] Berislav Buča, Joseph Tindall, and Dieter Jaksch, "Non-stationary coherent quantum many-body dynamics through dissipation," Nature Communications 10, 1730 (2019)

[17] Matthew A. Norcia, Matthew N. Winchester, Julia R. K. Cline, and James K. Thompson, "Superradiance on the millihertz linewidth strontium clock transition," Science Advances 2 (2016), 10.1126/sciadv.1601231

[18] Matthew A. Norcia, Robert J. Lewis-Swan, Julia R. K. Cline, Bihui Zhu, Ana M. Rey, and James K. Thompson, "Cavity-mediated collective spin-exchange interactions in a strontium superradiant laser," Science 361, 259-262 (2018)

[19] Helmut Ritsch, Peter Domokos, Ferdinand Brennecke, and Tilman Esslinger, "Cold atoms in cavity-generated dynamical optical potentials," Rev. Mod. Phys. 85, 553601 (2013).

[20] Markus Aspelmeyer, Tobias J. Kippenberg, and Florian Marquardt, "Cavity optomechanics," Rev. Mod. Phys. 86, 1391-1452 (2014). 
[21] Keye Zhang, Francesco Bariani, and Pierre Meystre, "Quantum optomechanical heat engine," Phys. Rev. Lett. 112, 150602 (2014).

[22] M Brunelli, A Xuereb, A Ferraro, G De Chiara, N Kiesel, and M Paternostro, "Out-of-equilibrium thermodynamics of quantum optomechanical systems," New Journal of Physics 17, 035016 (2015)

[23] A. Mari, A. Farace, and V. Giovannetti, "Quantum optomechanical piston engines powered by heat," Journal of Physics B: Atomic, Molecular and Optical Physics 48, 175501 (2015)

[24] Ken Sekimoto, "Kinetic characterization of heat bath and the energetics of thermal ratchet models," Journal of the Physical Society of Japan, Journal of the Physical Society of Japan 66, 1234-1237 (1997).

[25] Ken Sekimoto, "Langevin Equation and Thermodynamics," Progress of Theoretical Physics Supplement 130, $17-27$ (1998)

[26] Ken Sekimoto, Stochastic energetics, Vol. 799 (Springer, Berlin, 2010).

[27] Udo Seifert, "Stochastic thermodynamics, fluctuation theorems and molecular machines," Reports on Progress in Physics 75, 126001 (2012).

[28] R. Alicki, D. Gelbwaser-Klimovsky, and A. Jenkins, "A thermodynamic cycle for the solar cell," Annals of Physics 378, $71-87$ (2017)

[29] Alexandre Roulet, Stefan Nimmrichter, Juan Miguel Arrazola, Stella Seah, and Valerio Scarani, "Autonomous rotor heat engine," Phys. Rev. E 95, 062131 (2017).

[30] Stella Seah, Stefan Nimmrichter, and Valerio Scarani, "Work production of quantum rotor engines," New Journal of Physics 20, 043045 (2018)

[31] Klaus Hepp and Elliott H. Lieb, "Equilibrium statistical mechanics of matter interacting with the quantized radiation field," Phys. Rev. A 8, 2517-2525 (1973)

[32] Klaus Hepp and Elliott H Lieb, "On the superradiant phase transition for molecules in a quantized radiation field: the Dicke maser model," Annals of Physics 76, 360 -404 (1973)

[33] Peter Kirton, Mor M. Roses, Jonathan Keeling, and Emanuele G. Dalla Torre, "Introduction to the Dicke model: From equilibrium to nonequilibrium, and vice versa," Advanced Quantum Technologies 2, 1800043 (2019).

[34] Christoph Hamsen, Karl Nicolas Tolazzi, Tatjana Wilk, and Gerhard Rempe, "Two-photon blockade in an atomdriven cavity QED system," Phys. Rev. Lett. 118, 133604 (2017)

[35] Rahul Sawant and S. A. Rangwala, "Lasing by driven atoms-cavity system in collective strong coupling regime," Scientific Reports 7, 11432 (2017)

[36] Tony E. Lee, H. Häffner, and M. C. Cross, "Collec- tive quantum jumps of Rydberg atoms," Phys. Rev. Lett. 108, $023602(2012)$.

[37] G. Lindblad, "On the generators of quantum dynamical semigroups," Comm. Math. Phys. 48, 119-130 (1976).

[38] Vittorio Gorini, Andrzej Kossakowski, and Ennackal Chandy George Sudarshan, "Completely positive dynamical semigroups of N-level systems," Journal of Mathematical Physics 17, 821-825 (1976)

[39] H.P. Breuer and F. Petruccione, The theory of open quantum systems (Oxford University Press, 2002).

[40] Crispin Gardiner and Peter Zoller, Quantum noise (Springer, 2004).

[41] G. S. Agarwal, R. R. Puri, and R. P. Singh, "Atomic Schrödinger cat states," Phys. Rev. A 56, 2249-2254 (1997).

[42] Sarang Gopalakrishnan, Benjamin L. Lev, and Paul M. Goldbart, "Frustration and glassiness in spin models with cavity-mediated interactions," Phys. Rev. Lett. 107, 277201 (2011)

[43] See Supplemental Material for details.

[44] Mohammad Yaghoubi, M Ebrahim Foulaadvand, Antoine Bérut, and Jerzy Łuczka, "Energetics of a driven brownian harmonic oscillator," Journal of Statistical Mechanics: Theory and Experiment 2017, 113206 (2017).

[45] Hannes Risken, The Fokker-Planck Equation (Springer, Berlin, 1996).

[46] F. Benatti, F. Carollo, R. Floreanini, and H. Narnhofer, "Non-Markovian mesoscopic dissipative dynamics of open quantum spin chains," Physics Letters A 380, $381-389$ (2016).

[47] F Benatti, F Carollo, R Floreanini, and H Narnhofer, "Quantum spin chain dissipative mean-field dynamics," Journal of Physics A: Mathematical and Theoretical 51, 325001 (2018)

[48] Igor B. Mekhov, Christoph Maschler, and Helmut Ritsch, "Probing quantum phases of ultracold atoms in optical lattices by transmission spectra in cavity quantum electrodynamics," Nature Physics 3, 319-323 (2007).

[49] Ferdinand Brennecke, Stephan Ritter, Tobias Donner, and Tilman Esslinger, "Cavity optomechanics with a Bose-Einstein condensate," Science 322, 235-238 (2008).

[50] Kristian Baumann, Christine Guerlin, Ferdinand Brennecke, and Tilman Esslinger, "Dicke quantum phase transition with a superfluid gas in an optical cavity," Nature 464, 1301-1306 (2010)

[51] Nishant Dogra, Manuele Landini, Katrin Kroeger, Lorenz Hruby, Tobias Donner, and Tilman Esslinger, "Dissipation-induced structural instability and chiral dynamics in a quantum gas," Science 366, 1496-1499 (2019)

[52] Berislav Buča and Dieter Jaksch, "Dissipation induced nonstationarity in a quantum gas," Phys. Rev. Lett. 123, 260401 (2019) 